\title{
El mapa de Chile y el plano de Santiago en la obra atribuida a Juan Ignacio Molina de 1776: los manuscritos perdidos ${ }^{1}$
}

\author{
Rodrigo Moreno Jeria²
}

\begin{abstract}
RESUMEN
En la obra "Compendio della Storia geografica, naturale e civile del Regno del Chile" publicada en 1776 en forma anónima por el entonces ex jesuita Juan Ignacio Molina, se adjuntaron algunos mapas, planos y grabados, todos firmados por Giovanni Fabbri aunque atribuidos a Molina, en específico, un mapa de Chile, y un plano de la ciudad de Santiago. La razón de la atribución de Molina pese a no ser cartógrafo, se debe a que pudo ser el creador intelectual, coincidente por su condición de conocedor del espacio descrito, por lo que el nombre de artista, Fabbri, quien nunca estuvo en Chile, pasó a segundo plano. Sin embargo, un reciente hallazgo de nuevos manuscritos, permite descubrir que las referidas piezas, fueran realizadas por otro artista, Giussepe Mancini, quien supo representar tanto el territorio de la gobernación, así como su capital, siguiendo las instrucciones del sacerdote chileno, es decir, puso la ciencia cartográfica al servicio de la representación e imaginario de un intelectual que recordada su patria desde el exilio.
\end{abstract}

Palabras claves: Juan Ignacio Molina, Giuseppe Mancini, Giovanni Fabbri, Cartografía, Jesuitas.

\begin{abstract}
Included in the work "Compendio della Storia geografica, naturale e civile del Regno del Chile", published anonymously in $\mathbf{1 7 7 6}$ by the then ex-Jesuit Juan Ignacio Molina, were several maps, plans, and engravings. Although attributed to Molina, the illustrations, and specifically a map of Chile and a plan of the city of Santiago, were signed by Giovanni Fabbri. The reason for attributing them to Molina, despite his not being a cartographer, is due to his likely being the intellectual author of the work and coincides with his knowledge of the represented areas. As a result, the name of the artist, Fabbri, who had never been in Chile, receded into the background. However, a recent discovery of additional manuscripts has revealed that the aforementioned illustrations were created by a different artist, Giussepe Mancini, who was able to depict both the territory of the government and its capital, following the instructions of the Chilean priest. That is to say, Molina entrusted the science of cartography to the portrayal and imagination of an intellectual who remembered his homeland from exile.
\end{abstract}

Keywords: Juan Ignacio Molina, Giuseppe Mancini, Giovanni Fabbri, Cartography, Jesuits. 
Cuando se publicó el "Compendio della Storia geográfica, naturale e civile del Regno del Chile", editado en 1776, llamó la atención de los estudiosos la misteriosa autoría de una obra presentada como anónima. En un comienzo se pensó que el autor de dicho trabajo era del jesuita Felipe Gómez de Vidaurre, pero en el siglo XIX, con estudios como el de Diego Barros Arana (Barros Arana, 1886) se pudo afirmar con mayor certeza, que el anónimo escritor era nada menos que el abate Juan Ignacio Molina, el mismo que había publicado y firmado el "Saggio sulla storia naturale del Chili" en 1782 y el "Saggio sulla storia civile del Chili" publicada en 1787, y que mucho aportó desde el exilio en Italia al conocimiento de Chile y en particular de su historia natural dado que sus obras fueron traducidas y bien difundidas en diversos círculos académicos y bibliófilos. Posteriormente son numerosos los autores que han aceptado la autoría del abate, (Briones, 1968: 108-109; Ronan y Hanisch, 1979). Sin embargo, investigaciones recientes (Stuardo, 2007), ha vuelto a sostener que la obra de 1776 es de Gómez de Vidaurre, lo que demuestra que en la disciplina histórica es complejo dar por cerrada una controversia. Nosotros nos inclinamos con los fundamentos de quienes defienden la autoría de Molina.

De todas formas, por sus obras firmadas, que fueron publicadas desde la referida de 1782 hasta su "Memorie di storia naturale" de 1821, Juan Ignacio Molina, se transformó una figura relevante en la historia de Chile, haciendo desde el exilio una profunda promoción de su patria a la que nunca pudo regresar desde su forzosa partida de 1768.

Ahora bien, el centro de nuestra atención será la cartografía que se presentó en aquella obra anónima de 1776 y cuyo ejemplo más renombrado fue la publicación de un mapa de Chile (Ramírez, 1995) y plano de Santiago, que han sido considerados unos referentes en la cartografía histórica de Chile (Rosenblitt y Sanhueza, 2010). Recientemente, en una colección privada han aparecido dos piezas manuscritas que, datadas en torno a 1775, corresponden a los prototipos originales de la obra impresa. Y si el hallazgo es relevante, lo es más el hecho de que dichos manuscritos tienen por autor a un personaje desconocido a la fecha, Giuseppe Mancini, originario de Pesaro, con lo cual proponemos en este estudio, descartar fundamentadamente la autoría de Molina como autor de las piezas cartográficas y también demostrando que Giovanni Fabbri, firmante en las obras aludidas en el impreso, actuó solo como grabadista y se basó en un dibujo original que hoy se recupera. No obstante, la información que el cartógrafo tuvo para realizar las dos piezas, sólo pudo ser obtenida gracias al conocimiento e imaginario del territorio que tenía el religioso chileno, por lo que, tal como afirma Harley, estos mapas fueron fruto de una división de labores, en una transición que va desde el origen del manuscrito a la impresión (Harley, 2005).

\section{Molina: de jesuita a abate}

Pese a la importancia que representa Molina en la historia de Chile, considerado uno de los más destacados naturalistas de la Colonia (Briones, 1968; Rojas, 2001; Orrego, 2011; Charrier y Hervé, 2011) y calificado por algunos como el primer científico chileno (Saldivia, 2004), no son muchos los antecedentes biográficos que se conocen de su persona, aunque se explican fundamentalmente porque ingresó muy joven a la vida religiosa, y en el ámbito familiar, no quedaron muchos testimonios que sobrevivieran hasta nosotros.

Nacido el 24 de junio de 1740, en la hacienda de Huaraculén, actual comuna de Villa Alegre, región del Maule, pocas noticias tenemos de sus primeros años de vida, aunque sabemos que se 
formó en primera letras en el colegio de los jesuitas de Concepción (Briones, 1968), pero a los siete años de edad regresó a Talca tras la muerte de su padre (Briones, 1968). Allí continuó su formación hasta que a los 15 años optó por ingresar a la Orden de la Compañía de Jesús. La entrada al noviciado ocurrió el 12 de noviembre de 1755 (O’Neill y Domínguez, 2001), trasladándose a vivir a Santiago, residiendo en el noviciado San Francisco de Borja que se ubicaba en La Cañada. Luego, en 1757, tras su primera profesión, pasó a la Hacienda de Bucalemu, donde funcionaba también la casa de Tercera Probación, iniciando ahí sus estudios de humanidades y letras clásicas.

Siendo allí estudiante de retórica, como solían hacerlos los jóvenes jesuitas para ejercitarse en lengua latina, Molina escribió sus primeras elejías latinas (Castillo, 2016), y al parecer estudió italiano (Hanisch, 1974), dominio de la lengua que sería clave para emprender sus publicaciones de 1776, 1782 y 1787 (Rojas, 1965).

Terminada su etapa formativa en humanidades, pasó al colegio Máximo San Miguel en Santiago donde estaba estudiando teología hacia 1767, fecha del extrañamiento. De hecho, cuando fueron trasladados a Valparaíso, un testigo registra el hecho de que durante la espera de la nave que los llevaría al exilio, en el mes de enero de 1768 se tomaron los exámenes del curso de segundo de teología entre los que estaba el estudiante Molina, quien para entonces tenía 27 años de edad (Matthei y Moreno, 2001).

Lamentablemente para este joven estudiante, los jesuitas expulsos no estaban autorizados a llevar consigo ningún libro y documentos hacia el destierro, por lo que le fue confiscado un manuscrito en El Callao, y que tenía relación con una historia natural de Chile, aún en elaboración.

Trasladado al Puerto de Santa María y luego a Italia, específicamente a los Estados Pontificios, se instaló junto con el grupo de jesuitas expulsos de Chile en Imola, ciudad donde terminó sus estudios. Recibió la ordenación sacerdotal de manos del obispo de Bertinoro, Monseñor José Columbani y realiza su última profesión en Imola el 15 de agosto de 1773, cuando la Compañía de Jesús había dejado de existir oficialmente por el Breve apostólico del Papa Clemente XIV, Dominus ac Redemptor del 21 de julio de ese mismo año, sin embargo, este documento se hizo efectivo en la ciudad donde residía el 25 de agosto, es decir, Molina alcanzó a ser profeso solemne de cuatro votos por sólo 10 días (O’Neill y Domínguez, 2001; Hanisch, 1972).

Fue en ese momento en que pasó a ser "abate", puesto que, al dejar de ser jesuita, como sacerdote secular, se trasladó a vivir a Bolonia, ejerciendo el ministerio y al mismo dedicándose al estudio y enseñanza.

Bolonia era la célebre ciudad universitaria, sede del histórico Studium. Ahí puedo continuar su formación leyendo a autores que en América no tenía acceso y a otros que sí había conocido a través de la rica biblioteca del Colegio Máximo San Miguel de Santiago, y a los que tuvo acceso en sus 5 años que residió en Imola. Sin embargo, también comenzó a trabajar en la enseñanza, llegando a dictar clases en la Academia de Bolonia y dar cursos libres. Pero junto a ello, el interés que desde joven había mostrado por escribir, le animó a retomar sus estudios de historia natural, siendo la primera obra conocida, el citado Compendio anónimo de 1776, el que debió ser una reconstrucción de sus manuscritos perdidos en el extrañamiento, aunque sin posibilidad de cotejar información de la que disponía hasta principios de 1768. De hecho, Hanisch advierte que posiblemente los errores contenidos en esta edición, inhibieron a que el autor anónimo reconociera la autoría posteriormen- 
te, habiendo tenido muchas oportunidades para hacerlo, entre otras, cuando recuperó sus papeles y finalmente pudo publicar su primer Saggio de 1782 (Hanisch, 1972; Briones, 1968).

Junto con su segunda obra firmada, el "Saggio sulla storia civile del Chili" editado en 1787, el objetivo de Molina era publicar una traducción al castellano, dado el interés que había despertado su publicación. Pero la tarea no era fácil porque tenía que pasar por la censura hispana y como ex jesuita, el escenario no era propicio. Sin embargo, como bien lo demuestra Hanisch, se pudieron sortear todos obstáculos y el libro salió a la luz en 1788. (Hanisch, 1972). La traducción del Ensayo sobre la historia civil se concretó en 1795.

Los siguientes años continuó con sus trabajos, investigación y enseñanza. Recibió reconocimientos, pero también vivió algunos conflictos con su obra "Las analogías menos observadas en los tres reinos de la naturaleza", que le valieron injustamente una investigación de la que fue absuelto (O’Neill y Domínguez, 2001).

Con deseos de regresar a Chile nunca se pudo concretar dicho anhelo y falleció en Bolonia en 1829, siendo sepultado en el mausoleo de hombres ilustres de dicha ciudad, pero en 1966 sus restos fueron trasladados a Villa Alegre, en su tierra natal donde hoy descansan.

\section{La importancia del mapa de 1776 en el contexto jesuítico}

En el desarrollo científico del siglo XVIII, la cartografía tuvo un papel protagónico en cuanto a la representación y apropiación del espacio. Para el mundo político, los mapas fueron instrumentos de poder y por lo tanto, su elaboración se intensificó con el correr de los siglos, al mismo tiempo que progresaba la exploración, en especial de las periferias, y mejoraban las técnicas de representación, y se multiplicaban los progresos instrumentales.

Pese a ello, los mapas continuaban incorporando imaginarios, puesto que la apropiación de los espacios incluía las interpretaciones que no siempre coincidían con la realidad, es decir, pese a que los mapas podrían ser manifestación de las matemáticas, terminaban siendo "metáforas o símbolos del mundo" (Harley, 2005).

Las órdenes religiosas no estuvieron exentas de aquello. Los mapas tenían finalidades concretas que iban más allá de un fin científico. Se buscaba identificar el espacio con fines pastorales, y, además, colaborar con el orden establecido, como, por ejemplo, el dominio hispano del territorio en el caso chileno.

Por lo anterior, para representar un territorio se requería de testigos visuales que colaboraran en su representación, y para la Compañía de Jesús, los misioneros, o al menos, una parte, cumplieron dicho rol, convirtiéndose en voceros de información que posteriormente un cartógrafo pudo interpretar, aunque siempre bajo los parámetros de quien le daba las instrucciones, del mandante. Sin embargo, hubo casos en que el cartógrafo fue al mismo tiempo el misionero, y por supuesto que ahí el aporte a la ciencia cartográfica fue mucho más sustantivo.

En este contexto debemos entender el surgimiento de una cartografía asociada a la Compañía de Jesús en Chile (Moreno, 2013), de la cual hay ejemplos tempranos en el siglo XVII y los que 
referiremos para el siglo de la ilustración, al cual pertenece el mapa de Chile de 1776, el cual es un hito dentro de la historiografía jesuítica a tal punto que fue considerado en el siglo como el mejor que se había hecho hasta el momento (Hanisch, 1972; Ramírez, 1995). Y la verdad que mucha razón había, puesto que, hacia la fecha no eran muchas las contribuciones realizadas en el país.

De hecho, una buena parte de los mapas de Chile que circulaban hasta el momento, correspondían a adecuaciones a la Tabula Geographica publicada por Alonso de Ovalle en 1646, que al circular por Europa influyó en la cartografía que sobre Chile se realizó. Por ejemplo, los trabajos de Nicolas Sanson en 1657 y de Jacques-Nicolas Bellin en 1764 nos presentan mapas de Chile que fueron delineados en Europa sin conocimiento del territorio, y que terminaron siendo influenciados por la obra de Ovalle con adaptaciones posteriores, pero sin un cartógrafo que trabajara in situ en el territorio. Sin embargo, en la segunda mitad del siglo XVIII la labor de ingenieros militares como Juan Garland en Valdivia, nos muestran que, si uno de aquellos profesionales hubiese delineado Chile, los resultados hubiesen sido distintos a los que se conocían (Guarda y Moreno, 2010), tal como el intento realizado, o al menos firmado, por Ambrosio O 'Higgins en 1768 a propósito de la expulsión de los jesuitas, mapa que parece estar basado en alguno que realizó un miembro de la orden referida.

Por lo anterior, es efectivo que el mapa de 1776 publicado en el Compendio, puede ser considerado como uno de los mejores logrados hasta la fecha, puesto que no había buenos ejemplos, y los que existían, no abarcaban todos los territorios de la gobernación, o como en el caso del archipiélago de Chiloé, era evidente que existía una gran diferencia entre quienes representaban la isla por conocerla in situ, y los que simplemente se basaban en noticias de terceros, siendo los resultados diametralmente opuestos en cuanto a lo fidedigno de la información geográfica (Guarda y Moreno, 2008).

En cuanto a los jesuitas propiamente tal, no era mucho lo que habían aportado en cartografía durante los siglos XVII y XVIII, pero igual, en el contexto de ausencia de cartógrafos, fue importante para Chile Colonial. El mapa de Ovalle seguía siendo una referencia, pero con las carencias propias de su tiempo. Y el resto de la cartografía jesuítica, fundamentalmente del siglo XVIII apuntaba develar territorios específicos como el de Chiloé, y cuya mejor pieza es la realizada por los Padres Melchor Strasser y Michael Mayer (Moreno, 2013), aunque también el Padre Bernardo Havestadt realizó un mapa geográfico de un recorrido misional por la cordillera de los Andes (Meier y Müller, 2011). Y el referido Padre Gómez de Vidaurre delineó un mapa sobre Chiloé (Guarda y Moreno, 2008) el que, si bien fue realizado en el exilio, fue elaborado sobre la información de una carta de 1737 de inferior precisión cartográfica. Y sobre los territorios australes, el mapa del Padre José García, sobre un recorrido realizado al sur de Chiloé en 1766 y 1767, es una pieza de alto valor científico porque por representa territorios insulares que hasta esa fecha eran muy desconocidos para la ciencia cartográfica.

Sin embargo, hay un mapa de Chile perdido que debió ser el más importante realizado hasta la segunda mitad del siglo XVIII, o al menos, de lo que Molina vio o escuchó hablar. Se trata del mapa de Chile del Padre Juan Evangelista Fertl, un jesuita bávaro que trabajó en Chile desde 1725 en las misiones al sur del Bío Bío con mapuches (Meier y Müller, 2011). Llegó a ser superior de las misiones de Arauco, también fue rector del Colegio de Concepción. Con un gran conocimiento del territorio de la frontera, elaboró un mapa, el cual Molina refiere como el mejor en su tiempo, aunque lamentablemente hasta la fecha no se ha podido hallar (Meier y Müller, 2011). De hecho, 
Molina todavía esperaba tenerlo cuando preparaba la edición de 1810. El Padre Fertl falleció en Santiago el 1 de febrero de 1764, por lo que es probable que dicha pieza cartográfica permaneciera en Chile hasta su traslado con todos los documentos que se requisaron para la junta de temporalidades, aunque el mapa nunca más apareció.

Por lo anterior, el trabajo cartográfico de 1776 representaba la mejor muestra que hasta la fecha se hubiera realizado (Ramírez, 1995). Sin embargo, quedaba en evidencia que la ausencia de un cartógrafo, como sí los hubo en otras provincias de la Compañía en América, atentó para que la cartografía científica del siglo XVIII no tuviera un buen exponente en Chile, al menos hasta el extrañamiento de la Compañía a comienzos de 1768.

Los mejores ejemplos, Strasser y Mayer, Haverstadt y Fertl, nos indican que una buena formación matemática y científica en general, como la que recibieron estos cuatro misioneros germanos en su tierra, pueden ser coincidentes con que justamente ellos se animaron a realizar los referidos mapas y cartas.

Para los jesuitas, la cartografía en las misiones tenía un papel importante, puesto que no solo era fiel reflejo de la educación matemática promovida en sus colegios, sino también porque colaboraba activamente en la tarea de medir el mundo, puesto que, conociendo el espacio, y dominándolo geográficamente, el éxito de la misión podía venir por añadidura. (Romano, 2007).

Por todo lo anterior, identificado el valor del mapa "Il Chili Regno dell'America Meridionale" como uno de los más importantes, gestados en el entorno de la Compañía de Jesús, aunque con fines políticos y científicos no misionales, ahora es importante dilucidar quién fue el verdadero autor de dicha pieza cartográfica, así como también el plano de Santiago que también se publicó en el referido Compendio.

\section{El mapa de Chile de 1776: un nuevo manuscrito}

Así como desde el momento de la publicación del Compendio, por su condición de obra anónima, existieron dudas acerca de su autoría, que como señalamos, han persistido durante años, nunca hubo cuestionamientos sobre quien había realizado la bella pieza cartográfica titulada "Il Chile Regno Dell'America Meridionale" inserta en la obra. Se trataba de Giovanni Fabbri, un reconocido artista de Bolonia que firmó los grabados.

Ahora bien, habitualmente, la historiografía ha asociado al abate Molina la autoría de la representación cartográfica, porque se asume que el autor intelectual era el jesuita, y porque es muy habitual que los planos insertos en libros terminen siendo atribuidos al autor firmante de la obra en general. Pero como en el Compendio el libro fue anónimo, la atribución se produjo porque en el "Saggio sulla storia naturale del Chili" de 1782, Molina nuevamente incorporó el mismo mapa, y entonces desde ahí que la asociación que se hizo de esta pieza cartográfica con el ex jesuita exiliado en Bolonia, se convirtió en permanente.

Sin embargo, el hallazgo de un manuscrito de gran formato, posiblemente realizado en 1775, firmado por Giuseppe Mancini, originario de Pesaro, pero del que desconocemos ma- 
yores antecedentes biográficos hasta la fecha, obliga a revisar las convicciones que hasta entonces se tenían del creador de la carta geográfica, puesto que es evidente en el análisis comparativo del manuscrito y el impreso, que este último se hizo a partir del delineamiento de Mancini. Situación de toda lógica si pensamos que Fabbri no era cartógrafo y tuvo que hacer el mapa basándose en alguna obra. En algún momento se pensó en que pudo ser la influencia del mapa de Nicolas Sanson de 1657, pero en realidad este cartógrafo francés se basó a su vez en el de Ovalle de 1646.

Hallado en Italia por Barry Lawrence Ruderman y adquirido para la importante colección cartográfica de Juan \& Peggy Rada, esta joya de la historia de la cartografía chilena fue realizado con tinta y pluma y coloreado con pintura al agua.

En cuanto a su tamaño, el manuscrito es de gran formato, de 55,2 x 89,1 cm, en cambio en impreso firmado por Fabbri es de 32,4 x 43,7 cm. Y con respecto a la escala, Mancini la estableció de 180 millas geométricas y en el impreso de 1776 se indican 40 leguas.

Figura $\mathrm{N}^{\circ} 1$

Giuseppe Mancini, Il Chile Regno Dell'America Meridionale, c.1775.

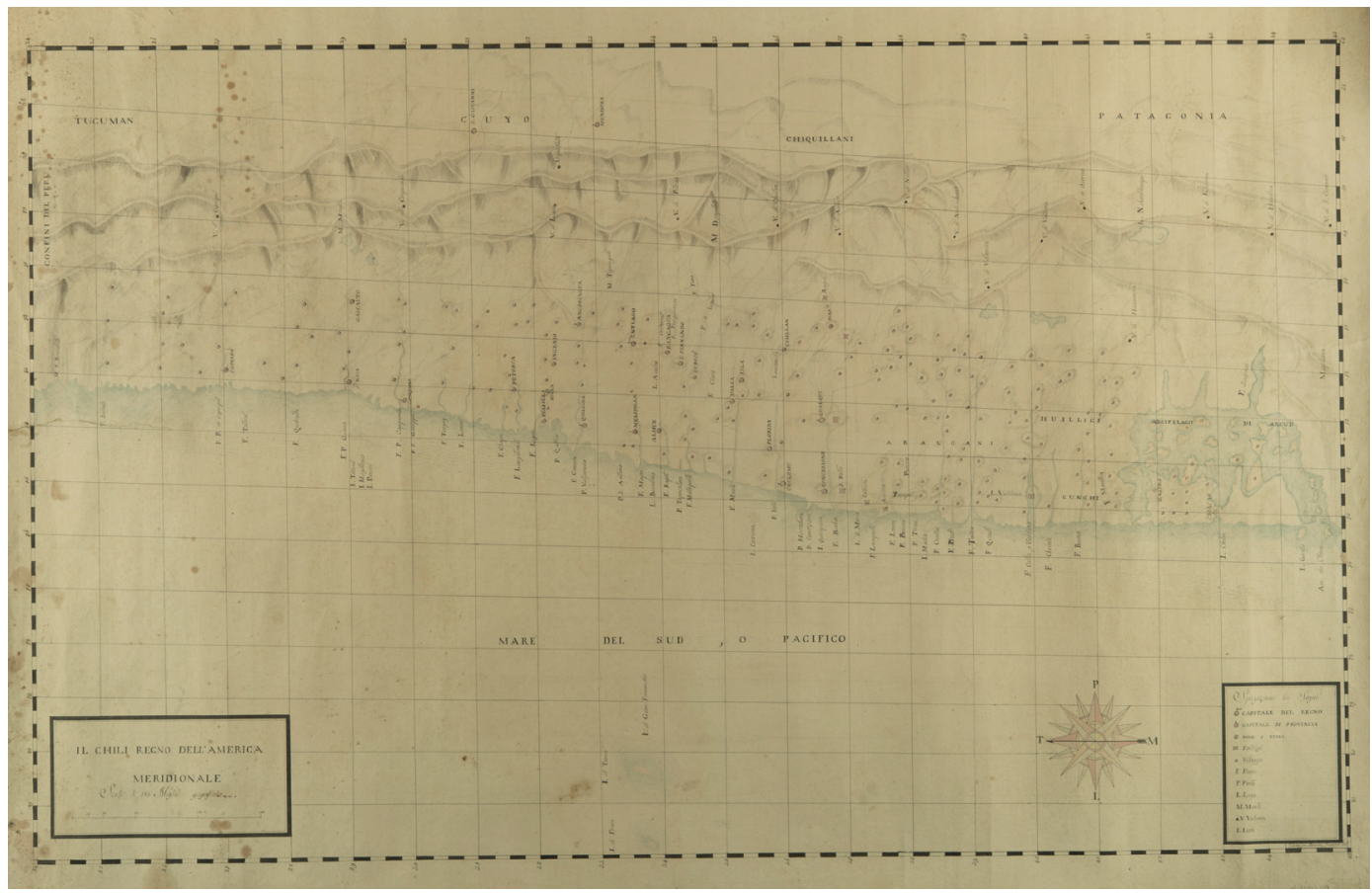

Sobre el contenido, la obra manuscrita posee información de interés. Los volcanes están indicados por sus nombres, a diferencia del texto impreso en donde hay algunos, particularmente en el sur en que figuran como anónimos. ¿Por qué? No lo sabemos, pero en el mapa en su versión de 1782 Fabbri corrigió la omisión. 
Por otra parte, el manuscrito de Mancini tiene un grave error en lo que corresponde a Chiloé. Invierte las posiciones de Castro y Chacao, confusión que Fabbri reparó posteriormente en el impreso. De igual forma, hay un error en el nombre del archipiélago al que se le llama Ancud, pero luego fue corregido en el impreso y se indica el topónimo correcto.

En general se puede observar que el manuscrito de Mancini está en castellano, en cambio Fabbri llevó varios topónimos al italiano, entre ellos el nombre de la capital, aunque la ciudad de Concepción se escribió en lengua itálica en las dos versiones.

En cuanto a los límites, Mancini especifica la frontera norte, que la indica con el Perú, en los 24 grados de latitud sur pero no hace referencia al límite en los 45 grados al sur del archipiélago de los chonos. En cambio, en el impreso, sí se indica que, en el sur, en el grado 46 se limita con la parte de la tierra magallánica.

Una diferencia que se observa entre ambos mapas, está en que el impreso extiende su representación hasta las sierras de Córdoba, en cambio en el manuscrito sólo se observa la región de Cuyo, pero sin la ciudad de San Luis. Posiblemente Molina u otro jesuita informante reparó en el error y le entregó mayor información sobre dicha provincia a Fabbri.

Por último, en la versión impresa se observa la notable representación artística de la leyenda, enmarcado en un cuadro finamente decorado y encabezado por un "indiano araucano", sin embargo, Mancini había sido mucho más austero y su recuadro, en la parte inferior derecha del mapa, solo indica el nombre y las escala en la que se trabajó.

Figura $\mathrm{N}^{\circ} 2$

Giuseppe Fabbri, Il Chile Regno Dell'America Meridionale, 1776.

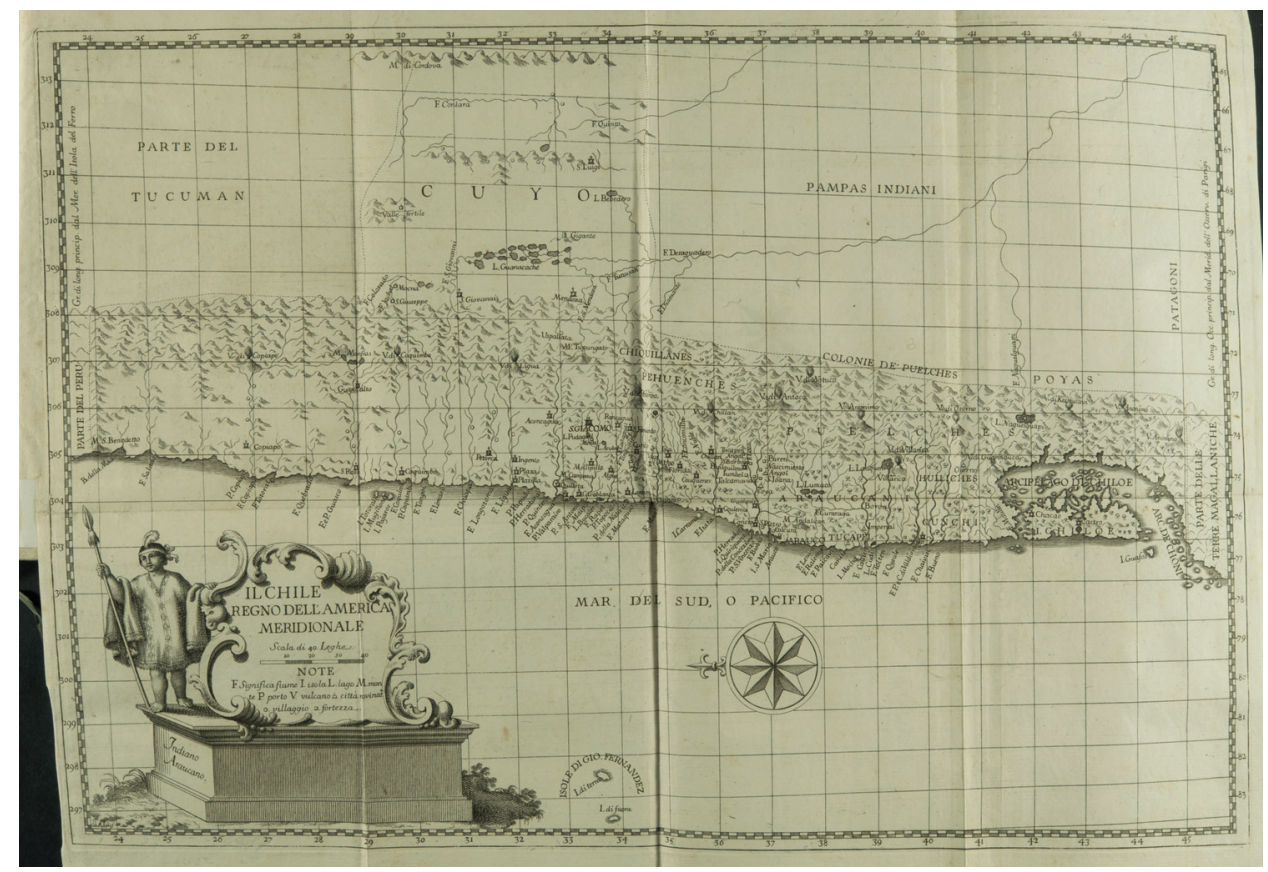


En definitiva, estamos frente a un mapa manuscrito que debió servir de prototipo de la versión final impresa en el Compendio. Mancini fue un autor que tuvo que trabajar a la ordenes de alguien que conocía bien el territorio chileno, y ese pudo perfectamente ser el mandante de la carta, el abate Molina.

El hallazgo de esta pieza cartográfica, junto el plano de Santiago del mismo autor, abren nuevas esperanzas de lo mucho que se puede avanzar en esta materia y del valor de este mapa de 1776 en la historia de la cartografía nacional.

\section{El Plano de Santiago: del Manuscrito de Mancini al grabado de Fabbri}

El bello plano de Santiago realizado por Mancini, es una pieza de gran formato, $45 \times 55 \mathrm{~cm}$., que contrasta con el impreso contenido en Compendio que solo tiene dimensiones más acotadas, llegando a 15,2 x 20,3 cm. Realizado con tinta y pluma, y coloreado con pintura al agua, fue hecho en escala de 3600 pies geométricos, que después Fabbri en el impreso redujo a 1350.

Figura $\mathrm{N}^{\circ} 3$

Giuseppe Mancini, S. Giacopo Capitale Del Regno Del Chile, c.1775.

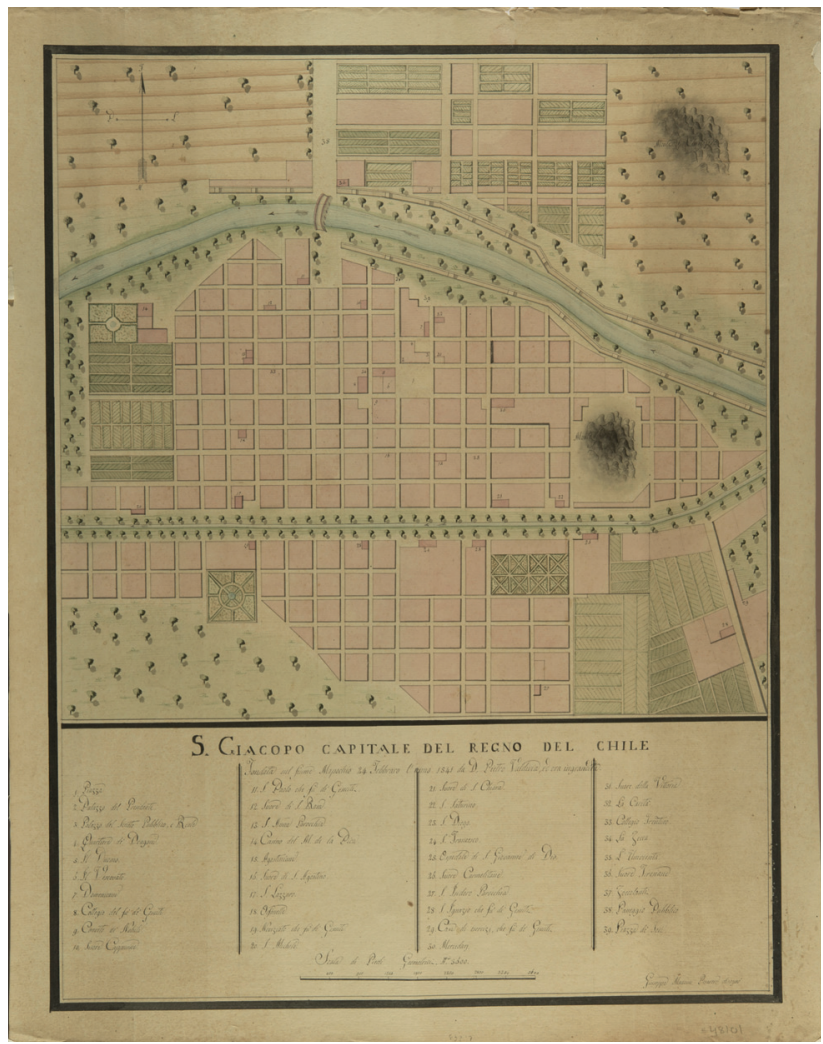


En cuanto al contenido del trabajo, el de Mancini está mucho mejor elaborado, con un manejo profesional que hace evidente la factura de un especialista en cartografía y planos. El encabezado y la explicación van en la parte inferior de la pieza, lo que permite extender los detalles en el barrio la Chimba, cosa que, en el Fabbri, lo imposibilita al haber agregado el título en la parte superior. Este detalle no es menor porque el crecimiento de la ciudad, que hacia 1776 tenía una población de 25.000 habitantes, se desarrollaba efectivamente hacia el sector norte, tal como lo demuestra la fundación de casas de religiosas como la Recoleta Franciscana fundada a mediados del siglo XVII, la Recoleta Dominica establecida en 1753 y el monasterio de las Carmelitas Descalzas que también se establecieron en la Chimba en 1770.

Ahora bien, hay una curiosidad interesante en el plano impreso de Fabbri, puesto que agrega dos nombres que son significativos y que están ausentes en el manuscrito. El primero es el topónimo "Mapocho" en el río, detalle que se podría deducir del propio título del plano de Mancini, pero que no lo hace explícito en la representación. En reemplazo de esa omisión, Mancini había agregado junto al título del plano lo siguiente: "Fondata sul fiume Mapochio". Pero en el impreso de Fabbri, el nombre del río está en español, y muy bien especificado (Figura $\mathrm{N}^{\circ} 4$ )

Además, hay un segundo topónimo que es toda una novedad. El autor del impreso identifica "La Chimba", conocido sector de Santiago que Mancini omite en el suyo. Lo anterior nos hace suponer que, para la confección del mapa definitivo, Molina pudo aportar este detalle o Fabbri lo copio de otros planos anteriores de Santiago como el de Ovalle (Ovalle, 1646), obra que debió estar disponible en Bolonia.

Y, además, queda claro que Fabbri sí tuvo acceso a dicha edición, porque en los grabados que adjunta al mapa y plano referidos, hay dos que claramente fueron tomados en su inspiración de la obra de Ovalle, específicamente el "Givoco la Chueca" y el "Ballo degl' Indiani" (Molina, 1776: tavola 5 y 7$)$.

En el caso del primero, la copia es casi exacta, aunque difieren en dos aspectos. Lo de Ovalle los danzantes están vestidos a usanza europea y con sombreros y plumajes, y en el caso de Fabbri, se acercan más a la fisonomía indígena, con cabezas descubiertas, aunque con vestimenta que también recoge influencia itálica. Además, el segundo aspecto, es que, en la edición de 1776, son 16 los danzantes siendo que en la obra de 1646 eran 18 los participantes (Ovalle,1646).

En cuanto a la segunda representación, las diferencias están en el número de participantes de la escena y el paisaje del entorno, muy detallado en Ovalle, y bastante simplificado por Fabbri, aunque manteniendo la misma estructura del dibujo e incluso, el número de chuecas o palos por parte de algunos indígenas quienes la tienen en su mano exactamente en la misma posición (Ovalle, 1646).

Un poco más distanciado de los dos ejemplos anteriores, pero guardando similitudes es el caso del dibujo de Fabbri titulado "Givoco il Quechucague", el cual si bien se inspiró en el publicado en Ovalle, titulado "Ludus Quechucague", en donde dos niños juegan al clásico "ludo" en versión mapuche, en la obra de 1776, consecuente con su mayor acercamiento científico, llevó al autor Fabbri, posiblemente con la ayuda del mandante Molina, a integrar en la representación tres joyas de la botánica chilena, la palma chilena o Jubaea chilensis (Molina, 1782); el "pino chileno", que para entonces aún no recibía su nombre definitivo de Araucaria o Araucaria Araucana (Moli- 
Figura $\mathrm{N}^{\circ} 4$

Giuseppe Fabbri, S. Giacopo Capitale del Regno del Chile, 1776.

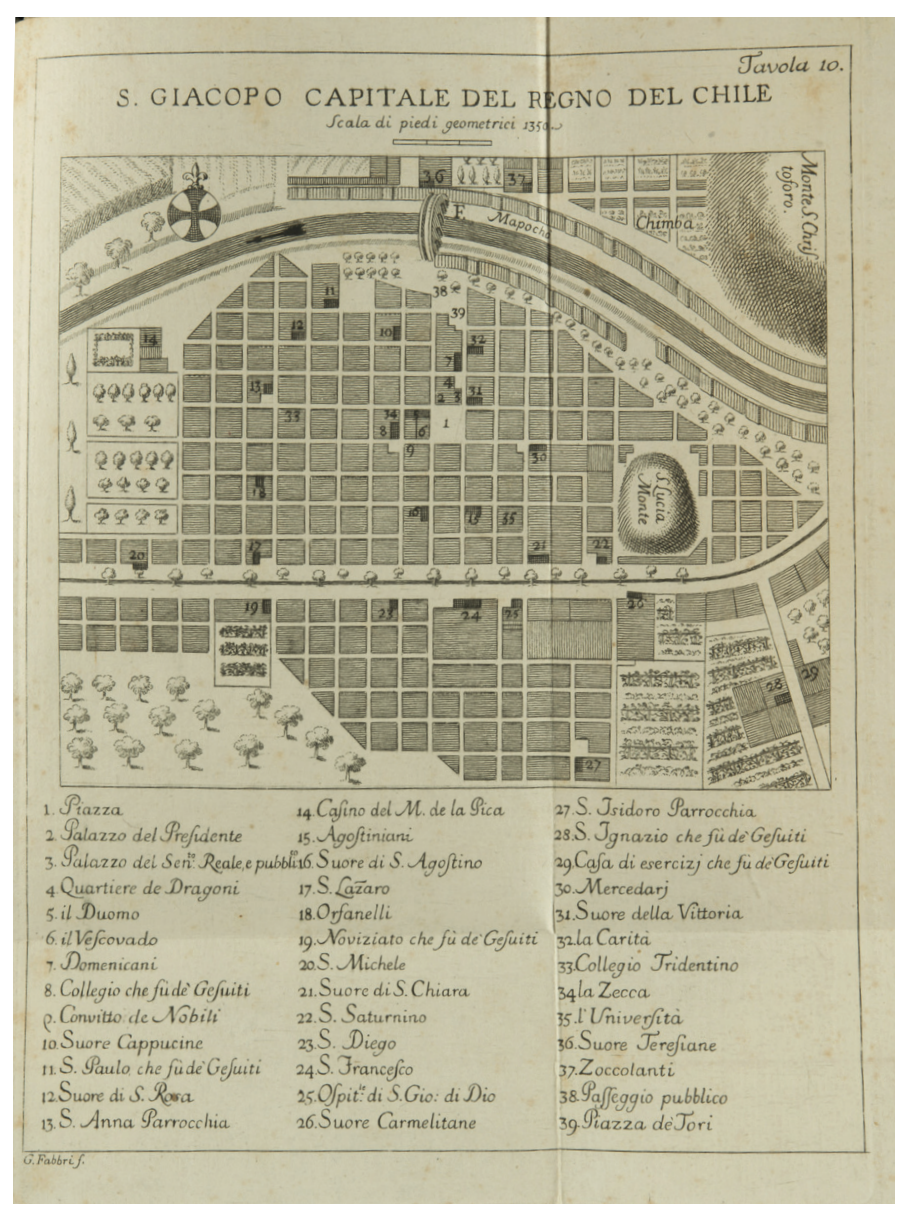

na, 1782) y el culén o Psoralea glandulosa (Molina, 1782), planta medicinal y comestible mapuche. En cambio, para el caso del dibujo publicado en 1646, el paisaje carecía de toda representación botánica (Ovalle, 1646).

En suma, la influencia de los grabados de la obra de Alonso de Ovalle, 130 años después de realizados por un autor anónimo y publicados en Roma, fueron replicados por el artista Fabbri, quien los complementó y actualizó con los antecedentes entregados presumiblemente por Molina para ilustrar su obra no firmada de 1776.

Por lo anterior, es posible que cuando el artista de Bolonia tuvo acceso al mapa de Ovalle, pudo rescatar información aplicable a su versión del Compendio, aunque queda en evidencia que el prototipo fue el plano de Mancini, y, además, con la presencia del propio Molina que tiene que haber verificado el resultado final. 
En cuanto a la lista que cada autor entrega sobre los topónimos a ubicar en el plano, coinciden los 30 nombres, por lo que ahí no hubo variación. Donde sí se observan pequeños detalles es, por ejemplo, en el ex noviciado de los jesuitas en La Cañada, identificado con el n¹9. En Mancini, en el huerto que dibuja en la parte posterior de la edificación que tenía su fachada hacia la vía principal, el autor quiso representar más bien jardines renacentistas que poco se acercaban a la realidad. En cambio, Fabbri simplifica el dibujo y lo hace más realista, seguramente a instancias de Molina que conocía muy bien el lugar.

En suma, el plano de Mancini se observa mucho más elaborado que el impreso, pero entiendo la gran reducción entre el tamaño del prototipo y el que finalmente resultó en el Compendio se puede entender que las modificaciones no alteraron la imagen a tal punto que, en una mirada rápida, se observan bastante similares.

Al igual que el mapa de Chile, este manuscrito de Mancini, fue hallado en Italia por la conocida casa de mapas antiguos Barry Lawrence Ruderman Inc., pasando recientemente a formar parte de la colección chilena de Juan \& Peggy Rada, radicada en la actualidad en Francia.

\section{Conclusiones}

Giovanni Fabbri habría delineado y grabado el mapa de Chile y el plano de Santiago en la obra anónima atribuida a Molina. Así queda claramente de manifiesto, porque dicho artista firmó explícitamente sus trabajos publicados en el Compendio.

Fabbri era una figura reconocida, de una larga trayectoria que se había iniciado en 1736 . Y precisamente en 1776, fecha de la publicación del mapa y plano, más las representaciones aludidas, fueron las últimas que realizó, puesto que falleció en Bolonia en enero de 1777. De hecho, sus biógrafos indican que el mismo año en que realizó el mapa y plano aludidos, hizo una reproducción del claustro octogonal de la iglesia de San Miguel in Bosco, una de sus obras más significativas. Sin embargo, Fabbri no era cartógrafo, por lo que la representación tan bien lograda en 1776, que se transformaría en un referente de la cartografía histórica chilena, solo superado en tiempos posteriores a la independencia por los trabajos científicos emprendidos por Claudio Gay (Rosenblitt y Sanhueza, 2010), debió haber sido realizada por alguien que tuvo conocimientos cartográficos, de la misma forma que Alonso de Ovalle los tiene que haber buscado cuando publicó su Tabula Geographica de 1646. Es decir, detrás de un autor intelectual, hubo un traductor de la realidad (Harley, 2005). Y tras ese traductor, hubo un representador, que definitivamente lo llevó a la impresión. Es decir, al menos tres personas participaron, el último de los cuales fue Fabbri.

Hacer mapas no era fácil, y menos aún, cuando se trataba de una carta general del territorio, y que, además, incluía precisiones técnicas que requerían condiciones de especialista. Es decir, si Molina tenía buenos conocimientos del territorio para alguien que era originario de Chile, eso no bastaba para realizar un mapa con este nivel de calidad. Entonces, se requería un cartógrafo o que tuviese formación como ingeniero militar, aspectos que son muy visibles en mapa firmado por Giuseppe Mancini.

¿Por qué Mancini es un autor tan desconocido pese a la calidad de su obra? ¿Por qué Fabbri no hizo alusión al verdadero delineador de la carta? Puede ser que Mancini, originario de Pesaro 
residiera en Bolonia y que haya un joven que trabajaba en el taller de Fabbri, quien hacia 1775 realizaba varios proyectos simultáneamente (Misiti, 1993). Quizás por ello, el maestro no requería citar a su discípulo y simplemente puso la rúbrica cuando el mapa de Chile, el plano de Santiago y resto de los grabados estuvieran listos. De ahí que incluso, para el caso de los grabados, el maestro no requirió prototipos, como lo que hizo Mancini, sino que se optó por recoger los grabados del libro de Ovalle y darles un sello particular como ya lo detallamos.

Ahora bien, queda claro que el originario de Pesaro sabía de cartografía, pero también se desprende del análisis del mapa y la carta, que no pudo haber realizado el mapa alguien que no conociera el territorio de Chile, y ahí entra en juego Molina, el mandante, quien debió realizar una exhaustiva revisión del manuscrito, que sirvió de prototipo para que el maestro Fabbri hiciera la versión final. Es decir, en la representación espacial de territorio, se conjugó la realidad vivenciada por Molina y su espíritu humanista (Jiménez, 1974), la interpretación de Mancini y el arte de Fabbri. De todas formas, hubo errores, como el que advertimos en Chiloé, pero fueron corregidos en la versión final lo que nos hace suponer que Molina, debió hacer revisiones antes de que el Compendio saliera de la imprenta.

Como hemos observado, en algunos aspectos los manuscritos poseen mayor información toponímica, pero en otros, se puede constatar que se aprovechó la mano de Fabbri para hacer precisiones y mejoras que finalmente llegaron a la edición definitiva.

Queda claro entonces que la aparición de estos dos manuscritos, vienen a transformarse en un significativo aporte al conocimiento de la cartografía y representación tardo colonial de Chile, y cuya curiosa coincidencia, es que fueron elaborados en Italia por autores locales, pero con la supervisión de un chileno. Si eso había ocurrido en 1646 con Alonso de Ovalle, ahora nuevamente se observa el fenómeno con Molina. Y lo que aún llama la atención en que, entre muchos especialistas, es que, se sigue hablando de los mapas de Ovalle y Molina, como si ellos hubiesen sido, con seguridad, los delineadores de dicha cartografía. Para el caso de Ovalle, persisten las dudas, pero para el caso de Molina, estos manuscritos solucionan una problemática: ahora conocemos los borradores y está firmados, es decir, Giuseppe Mancini fue el autor de "Il Chile Regno Dell'America Meridionale" y de "S. Giacopo Capitale del Regno del Chile". Sin embargo, el autor intelectual, hasta ahora sigue siendo el abate Molina.

\section{Referencias bibliográficas}

ANÓNIMO. Compendio della storia geografica, naturale, e civili del regno del Chile. Nella stamperia di S. Tommaso D’Aquino. Bologna, 1776.

BARROS ARANA, D. Historia General de Chile. Santiago de Chile: Rafael Jover, 1886.

BRIONES, H. El abate Juan Ignacio Molina: ensayo crítico-introductorio a su vida y obra. Santiago de Chile: Editorial Andrés Bello, 1968.

CASTILLO, M. El abate Molina y su poema "sobre los ríos de Chile". Anales de Literatura Chilena, 2016, No26, p. 99-113. 
CHARRIER, R. y HERVÉ, F. El abate Juan Ignacio Molina: una vida dedicada a la historia natural y civil del reino de Chile. Revista de la Asociación Geológica Argentina, 2011, Vol. 68, № 3, p.445-463.

GUARDA, G. y MORENO, R. Monumenta Cartographica Chiloensia. Santiago de Chile: Corporación del Patrimonio Cultural de Chile, 2008.

GUARDA, G. y MORENO, R. Monumenta Cartographica Valdiviensae. Santiago de Chile: Corporación del Patrimonio Cultural de Chile, 2010.

HANISCH, W. Historia de la Compañía de Jesús en Chile. Santiago de Chile: Editorial Francisco de Aguirre, 1974.

HANISCH, W. Itinerario y pensamiento de los jesuitas expulsos de Chile. Santiago de Chile: Editorial Andrés Bello,1972.

HARLEY, J.B. La nueva naturaleza de los mapas. México: Fondo de Cultura Económica, 2005.

JIMÉNEZ, J. El abate Molina: humanista clásico y sabio cristiano. Santiago de Chile: Anales de la Facultad de Teología N²4, Universidad Católica de Chile, 1974.

MATTHEI, M. y MORENO, R. Cartas e Informes de Misioneros jesuitas extranjeros en Hispanoamérica, quinta parte (1751-1778). Santiago de Chile: Anales de la Facultad de Teología, Pontificia Universidad Católica de Chile, 2001.

MEIER, J. y MÜLLER, M. Jesuiten aus Zentraleuropa in Portugiesisch - und Spanisch - Amerika. Ein bio-bibliographisches handbuch. Band 2: Chile (1618-1771). Münster: Aschendorff Verlag, 2011.

MISITA, M.C. Dizionario biografico degli italiani. Roma: Istituto della Enciclopedia italiana, Vol. 43, 1993.

MOLINA, J.I. Saggio sulla storia naturale del Chili. Nella Stamperia di S. Tommaso d'Aquino. Bologna, 1782.

MOLINA, J.I. Saggio sulla storia civile del Chili. Nella Stamperia di S. Tommaso d'Aquino. Bologna,1787.

MORENO, R. El Archipiélago de Chiloé y los Jesuitas: el espacio geográfico para una misión en los siglos XVII y XVIII. Magallania, 2011, Vol.39, N², p. 47-55.

MORENO, R. La cartografía jesuita en el archipiélago de Chiloé en los siglos XVII y XVIII. En: CASTRO SANTA MARÍA, A. y GARCÍA NISTAL, J. La Impronta Humanística (ss. XV -XVIII), Saberes, Visiones, Interpretaciones, Parlermo: Officina di Studi Medievali, 2013, p. 325-334.

O’NEILL, CH. y DOMíNGUEZ, J. Diccionario Histórico de la Compañía de Jesús. Biográfico - Temático. Madrid: Institutum Historicum S.I. - Universidad Pontificia Comillas, 2001. 
ORREGO, F. El "Mundus Subterraneus" de Juan Ignacio Molina o el geólogo como economista. Asclepio, 2015, No 67, p.112.

ORREGO, F. Juan Ignacio Molina y la Comprensión de la Naturaleza del Finis Terrae. Un acercamiento desde la Historia (Cultural) de la Ciencia. Arbor, 2011, N0187 -756, p. 961 - 976.

OVALLE, A. Historica relatione del Regno di Cile. Roma: Apresso Francesco Cavalli, 1646.

RAMÍREZ, H.R. Galería geográfica de Chile. El Abate Juan Ignacio Molina y Opazo: Su Descripción del Reyno de Chile. Revista de Geografía Norte Grande, 1995, N²2, p.111-115.

ROJAS, M. El Abate Molina y su obra. Anales de la Universidad de Chile, 1965, Vol. 133, № 73, p. 5-24.

ROJAS, M. El Fin del Milenio y el Sentido de la Historia. Manuel Lacunza y Juan Ignacio Molina. Santiago de Chile: LOM, 2001.

ROMANO, A. Actividad científica y Nuevo Mundo: el papel de los jesuitas en el desarrollo de la modernidad en Iberoamérica. En: MARZAL, M. y BACIGALUPO, L. (editores). Los jesuitas y la modernidad en Iberoamérica. Lima: Fondo editorial Pontificia Universidad Católica del Perú - IFEA - Universidad del Pacífico, 2007, p. 56-71.

RONAN, CH. y HANISCH, W. Epistolario de Juan Ignacio Molina S.J. Santiago de Chile: Editorial Universitaria, 1979.

RONAN, CH. Juan Ignacio Molina: the world's window on Chile. New York: Peter Lang, 2002.

ROSENBLITT, J. y SANHUEZA, C. Cartografía Histórica de Chile, 1778-1929. En: Cartografía Histórica de Chile. Santiago de Chile: Biblioteca Fundamentos de la Construcción de Chile, Santiago de Chile, 2010, p. ix- Ixvi.

SALDIVIA, Z. Juan Ignacio Molina, primer científico chileno. Humanidades en foco. Disponible en Internet: http://terra.cefetgo.br/cienciashumanas/humanidades_foco/anteriores/humanidades_2/ textos/molina.pdf, 2004.

STUARDO, J. Trascendencia del primer Saggio sulla storia naturale del Chili de J.I. Molina, su traducción, el Compendio Anónimo y el Bicentenario. Atenea, 2007, № 495, p. 83-107. 
